International Journal of Agriculture, Environment and Bioresearch

Vol. 06, No. 06; 2021

ISSN: $2456-8643$

\title{
MANAGING THE ONCOLOGICAL PAIN IN DOG AND CATS
}

\author{
Ruxandra Costea \\ University of Agronomic Sciences and Veterinary Medicine of Bucharest, 59 Marasti Blvd, District 1, Bucharest, \\ Romania \\ https://doi.org/10.35410/IJAEB.2021.5692
}

\begin{abstract}
In veterinary medicine, we face many barriers for appropriately managing cancer, and more specifically the pain associated with cancer. This article reviews the possibilities for managing pain in oncologic patients, the different ways to assess their pain and the advantages and disadvantages of the several options for pain relief. The biggest obstacle that appears when trying to manage cancer pain in companion animals is diagnosing the pain before it's too late, due to its terminal characteristics. Despite the therapeutic options, oncological or cancer pain remains an obstacle for patient's comfort and welfare during their illness. Practitioners need to recommend and treat cancer pain in a multimodal individualized way, by pharmacological or physical agent modalities. The management of oncological pain in veterinary medicine raise complex concerns over recognition, assessment and treatment and needs further researches in order to establish standardized protocols.
\end{abstract}

Keywords: Pain; Cancer; Analgesia, Veterinary.

\section{INTRODUCTION}

Cancer is a frequent diagnosis and an important mortality factor in actual veterinary medicine [1]. The incidence of cancer continues to increase in companion animals. Despite the importance of this increasing occurrence and the large number of studies performed, researches in this area are impede by the complexity of cancer [2].

Pain is described as "an unpleasant sensory and emotional experience associated with, or resembling that associated with, actual or potential tissue damage," [3]. Pain is a sensory phenomenon triggered by different types of stimuli that can generate a complex pain response [4]. Oncological pain represents an important clinical manifestation that influences directly the life quality and expectancy and aggravate the general prognosis. Pain will depend on the tumor type, size, localization or invasion of adjacent tissues and metastasis and has a highly individual manifestation frame. Pain assessment and therapeutic protocols will be modulated according to the individual response.

\section{PHYSIOLOGY OF PAIN}

In order to perform an effective pain management from assessment to treatment, it is vital to understand the physiology of pain. Between the initial tissue trauma until the pain responses, a succession of four events follows: transduction, transmission, perception and modulation (Figure 1) [5]. Transduction is explained by the change of somatic/visceral nociceptors stimuli into an action potential that will advance toward the cerebrum (transmission). In the dorsal horn of the 
spinal cord the noxious information is attenuated or amplified trough modulation and when the stimulus reaches the cerebral cortex the perception of pain develops [6].

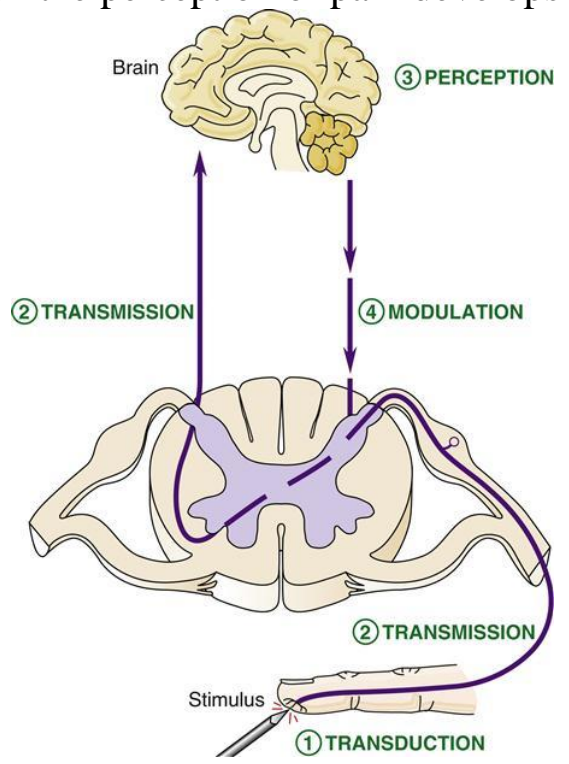

Figure 1. Four processes of pain signaling [7]

Acute pain generally accompanies a tissue damage and generates a response proportional to the injury [8]. On the contrary, chronic pain lasts for longer periods and is classically associated with a chronic inflammatory disease or a degenerative condition, or generated following nerve injury or damage. For the chronic pain the neuroendocrine response is attenuated. Acute pain has a good response to analgesics compared to the chronic pain. Chronic pain is described as resulting from persistent noxious factors acting in the periphery and determining changes at the level of dorsal spinal horn and/or central nervous system structures [9].

Types of pain may be classified as nociceptive or physiologic pain, inflammatory and neuropathic. Cancer pain may show the characteristics of both inflammatory and neuropathic pain [8]. Neuropathic, usually chronic pain caused by a lesion or a disease affecting the somatosensory system [10] is common in cancer. It can be determined by direct damage of the nervous system caused by a primary tumor or by metastases as well as from specific therapeutical actions, as chemotherapy [11]. Neuropathic pain can be persisting continuously or may determine repeated painful episodes [12]. Nociceptive pain arises from a damage to a nonneural tissue and is related to the activation of nociceptors [13]

The perception of pain starts from an external stimulus that stimulates the nociceptive sensory system. There is a specific pattern for which this noxious (unpleasant) stimulus is transmitted among the anatomical nociceptive pathway [14]. The free endings of primary sensory neurons are represented by receptors called nociceptors founded throughout the entire body and stimulated by mechanical, thermal or chemical factors.

There are two main types of nerve fibers which transport information from the nerve endings: primary afferent unmyelinated $\mathrm{C}$ fibers and myelinated A- $\delta$ fibers. Unmyelinated $\mathrm{C}$ fibers are those activated by intense stimuli, conducting an impulse slowly and under normal conditions have no background discharge. Unmyelinated $\mathrm{C}$ fibers respond to different stimulus because they are poly modal. More specifically they are not all nociceptors, some will respond to 
Vol. 06, No. 06; 2021

ISSN: $2456-8643$

noxious stimulus, and some may respond to lower temperatures, or even stroking of haired skin. Their threshold of stimulation is higher than those of other types of afferent sensory fibers, therefore they will need stronger stimulus. $\mathrm{C}$ fibers may be classified in peptidergic fibers, that release neuropeptides, and non-peptidergic [15].

A- $\delta$ myelinated fibers represent the primary sensory neurons free endings, described as type I and respectively type II. Type I is actually called A- $\delta$ fibers and will transmit the noxious sensory stimuli, and type II being A- $\beta$ fibers will transmit the non-noxious sensory information. Under abnormal conditions, and after tissue trauma, the physical properties of theses nociceptors change, and these A- $\beta$ fibers may also transmit noxious stimuli. Unmyelinated $\mathrm{C}$ fibers contribute to a slight burning sensation, whereas $A-\delta$ fibers conduct impulses quicker and favorize a powerful acute pain response [16]. Nociceptors can also be distinguished according to their differential expression of channels that analyze sensitivity to heat, cold, acidic environment and a range of chemical irritants [17]. These different classes of nociceptors are associated with specific function in the detection of distinct pain modalities.

\section{ONCOLOGICAL PAIN}

Oncological or cancer pain can be characterized as acute or chronic pain and separately as a mix of both nociceptive and neuropathic pain [18]. Toxins or inflammatory processes that are related to cancer can also contribute to pain manifestations [19]. Pain assessment for oncological cases should take into account different factors involved in the developing of the underlying disease, in order to define de pain in terms of etiology, different clinical manifestations and physiopathology pathways [20,21]. The infiltration of tumor cells and the peritumoral inflammation leads to direct tissue injury, which is perceived as nociceptive pain. The injured tissue will stimulate the primary neuron receptors within different structures and lead to the sensation of pain. Pain induced by the presence of cancer can also be determined by the invasion of metastatic tumor cells into different types of tissues at the peripheral nervous system level or the central nervous system [22]. The infiltration of tumors will determine visceral pain, perceived when the tumor determines compression and distortion of other internal organs situated in the abdominal, thoracic, or pelvic cavities. The pain is often manifesting as focal and acute pain similar to the sensation described in human medicine studies. The somatic and visceral pain syndromes can be characterized as nociceptive in nature. And ultimately, when the cancer cells infiltrate peripheral nerves, nerves plexus, spinal cord, the sensation is associated to neuropathic pain, which is described by human cancer patients as burning, shooting, pins/needles [15,23].

Since cancer pain will display the characteristics of both inflammatory and neuropathic pain, one single tumor can present both characteristic as a neuropathic and inflammatory pain as well [24].

\section{PAIN ASSESSMENT}

Since animals can't communicate directly their sensation and the level of pain, important alternative methods for identifying pain and its magnitude are crucial. The first and most important step for recognizing pain is the discussion with the owner. Frequent behavioral changes observed by pet owners could represent pain manifestations and include changes in movement, posture, grooming habits, changes in appetite, water consumption, focal licking, 
drooling, dysphagia, vocalization, respiratory frequency, defecation or urination habits modified [25].

For optimal pain management in animals, and for choosing the correct method to treatment, the veterinarian must be aware of numerous pain-scoring systems, based on specific behavioral and physiological responses as 'University of Melbourne Pain Scale", "Glasgow Composite Pain Tool", "Colorado State Acute Pain Scale" [26]. The assessment of cancer pain, can be done through pain scales as talked previously, however since they usually relate to either acute, or chronic pain, their correlation with cancer pain might be restricted. As a consequence, although cancer pain is recognized in animals, the ability to manage it is very limited [27]. Vital parameters (heart rate, respiratory rate, body temperature, pupil size, blood pressure) have been used to monitor patients and to asses pain in veterinary medicine, but without a strong specificity, since they are also influenced by multiple psychologic and stress factors [28]. Newer approaches developed for the assessment of pain include power spectral analysis of electroencephalogram. A quantified analysis of the electroencephalogram is performed while the animal is exposed to different noxious stimuli [5].

Starting from the necessity of having a dedicated tool for oncological pain assessment for small animals, along with the importance of owner's collaboration during the cancer therapy, a cancer pain inventory was developed for pet owners: Purdue Integrated Cancer Pain Score System, consisting in a Canine/Feline Brief Pain Inventory (Figure 2).

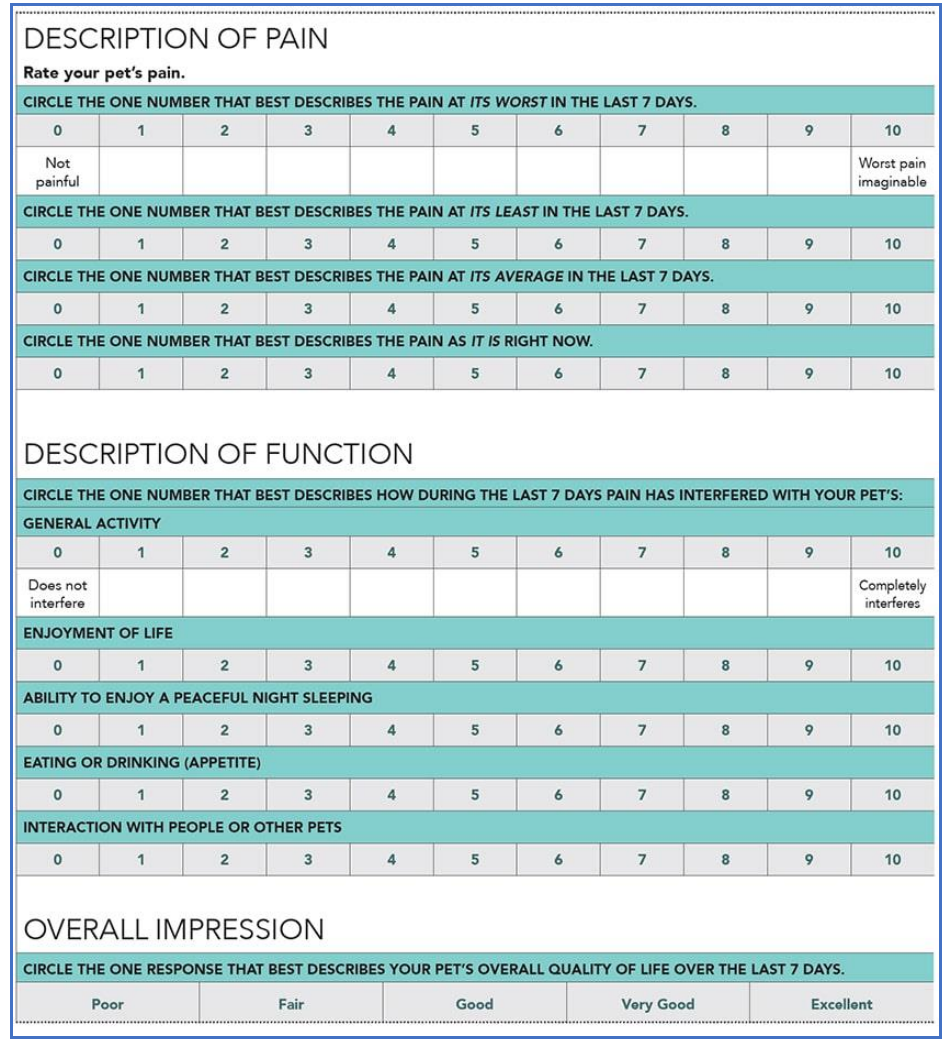

Figure 2. Modified Canine/Feline Brief Pain Inventory [29]. 
Vol. 06, No. 06; 2021

ISSN: $2456-8643$

\section{PHARMACOLOGICAL MANAGEMENT}

Many different analgesic pharmaceuticals can determine analgesia, by targeting pain transmission mediators and inhibiting the nociception pathway [30]. The multimodal individualized pain management, is based on the use of a combination of drugs with different mechanisms of action, decided according to each patient and each type of pain. It is used frequently for persisting pain, since managing such conditions in a simple pharmacological approach can be difficult and with a poor efficacity. The choice of the protocol depends on the severity of pain, type and localization, as well as on the clinical condition of the animal. Analgesic options include opioids, non-opioids or adjuvant, used alone or in different combination. Effective opioid treatment relays on several factors such as the appropriate drug selection, route of administration, dose, management of side-effects. The use of non-steroidal anti-inflammatory drugs in multimodal analgesia protocols, alone or combined with an opioid treatment can represent a method we can rely on for pain control, but the side effects should be weighed against their benefits and discussed on an individual level [20].

Opioids are an adequate option to use alone or in combination with other medications to inhibit pain, offering both analgesia and sedation. Therefore, opioid drugs will be used for treating severe pain. They exert their effect through the four primary types of receptors. $\mathrm{Mu}(\mu)$ receptors located supraspinal produce analgesia, sedation, euphoria, respiratory depression and determine addiction. Kappa $(\kappa)$ receptors located at the spinal level produce local analgesia and sedation. Delta $(\delta)$ receptors are located both spinally and supraspinal and produce only analgesia. The last group of receptors, sigma $(\sigma)$ has an unknown significance and may not be recognized by some investigators. In order to inhibit pain, they will be used at low doses. But when used at higher doses they will lead to sedation or even anesthesia. The side effects of opioids include respiratory depression, bradycardia, low gastrointestinal motility, nausea and vomiting or constipation. One of their advantages is represented by the possibility to reverse their action using naloxone. Opiates may manifest their effects from a few minutes until several hours, depending on the drug, dose and administration way selected. The numerous routes of administration make up multiple advantages in different species [31].

Non-steroidal anti-inflammatory drugs may be used for treating mild until moderate pain but their distinct disadvantage is the risk of adverse effects. The use of NSAID's to manage cancer patients is well supported. Not only because they are the fundamental drugs in controlling pain in veterinary medicine, but they also exhibit anti-tumoral properties. Many tumors, particularly carcinomas, over-express COX-2, which has been linked to their metastasis, immune suppression and angiogenesis capabilities. Therefore, treating these types of cancer-related pain, with a COX2 inhibitor, can be beneficial [32,33]. This group of analgesics inhibit the formation of prostaglandins and thromboxane, from arachidonic acid. Non-steroidal anti-inflammatory drugs inhibit cyclooxygenase enzyme from the arachidonic acid metabolism (COX-1 and/or COX-2), and determine anti-inflammatory effects along with antipyretic and analgesic ones, depending on the product used. Analgesia will be obtained by the reducing of the sensitization of nociceptors. Non-steroidal anti-inflammatory drugs will be used with precaution since they may lead to gastro-intestinal side effects or nephrotoxicity. Used for analgesia they have the advantage of not reducing the consciousness state or causing respiratory depression as opioids will determine [34].

Corticosteroids are essentially used to manage chronic pain. Overall, they decrease inflammation and prevent immune-mediated responses by entering the cell nucleus and binding 
Vol. 06, No. 06; 2021

ISSN: $2456-8643$

to chromatin in order to alter the RNA synthesis and to inhibit the prostaglandin cascade. They will suppress the white blood cell function. Their long list of adverse effects often limits their usefulness in pain management [36]. Glucocorticoids are used in combination with chemotherapy protocols for relevant tumors, and may also offer sufficient pain inhibition when administered. Their lymphocytotoxic and anti-inflammatory properties make them beneficial to patients with diseases such as lymphoma or mast cell tumors [36].

Ketamine is a N-Methyl-D-aspartate (NMDA) antagonist receptor, that can antagonize these receptors and will inhibit pain, preventing central sensitization. The complex structure of this receptor arranges multiple sites for therapeutic inhibition allowing for competitive and noncompetitive NMDA antagonists to bind the glutamate site [37]. The pharmacokinetic properties of ketamine after binding, it will decrease the time and periodicity the calcium channels are open, and therefore it will reduce the overall calcium ion concentration.

Unlike ketamine, amantadine will inhibit the NMDA response completely. Amantadine may be an advantage for patients with neuropathic chronic pain [38]. Amantadine, initially an antiviral agent against influenza it is used in human medicine as an analgesic in chronic cancer pain treatment protocols. Amantadine is most commonly used to treat drug reactions that affect coordination (extrapyramidal reactions) and pain in dogs. Side effects are may include agitation or diarrhea [39].

Gabapentin is an anti-convulsant used as a first-line therapy for the treatment of neuropathic pain. Its mechanisms and sites of action are not totally elucidated. Starting from the clinical results, the spinal and supraspinal sites it is believed that are modulating different aspects related to its effect in pain control [40].

Alpha2 agonist drugs provide analgesia by binding to pre-synaptic alpha2 receptors, and thus decreasing the release of norepinephrine, which result in decreased formation of pain. They are located at different anatomical regions of the body like the locus coeruleus in the brain, the dorsal horn of spinal cord and at the sympathetic nerve endings [4,41]. An example of the new generation alpha2 agonist is dexmedetomidine with similar analgesic and sedative effects and less cardiovascular effects. Dexmedetomidine is twice as potent as medetomidine and will be used in micro doses [42]. Alpha2 agonists may cause bradycardia secondary to increased vagal tone and are contraindicated in patients negatively affected by a decreased cardiac output and increased afterload [43].

Local anaesthetics can inhibit pain by blocking the depolarization of neurons and their ability to transmit information. This agent suppresses action potentials in excitable tissues by blocking voltage-gated $\mathrm{Na}+$ channels. In doing so, they inhibit action potentials in nociceptive fibers and so block the transmission of pain impulses [44]. A very effective method for the preemptive analgesia is the administration of local anesthetic agents; blocking the nerve impulse transmission before reaching the second and third nerve fibers within the pathway [45]. Analgesic drugs used for premedication (opioids, alpha2 agonists, NSAIDs) also provide preemptive analgesia which decreases central sensitization and total amount of analgesics needed as well as helps mitigate the postoperative pain experience [46].

Bisphosphonates have been shown to reduce the worsening of pain associated with bone metastases in patients with advanced solid tumors. Bisphosphonates may delay the progression of pain associated with bone metastases in patients with advanced cancer [47]. 
Vol. 06, No. 06; 2021

ISSN: $2456-8643$

The discovery of cannabinoid receptors and endocannabinoid system has led to the potential therapeutic use of cannabis derivatives. Cannabinoids acting through the CB1 receptors modulate the release of other neurotransmitters in the central nervous system, whereas the activation of peripheral CB2 receptors results in decreased inflammatory response and increased apoptosis of some tumor cell populations [48]. The stimulation of cannabinoid receptors (CB1 and CB2) was discovered as a catalyst for reducing neuropathic and inflammatory pain, and also reducing tumor growth and angiogenesis in some types of cancers. The activation of CB2 receptors reduce inflammation and the progression of atherosclerosis, while increasing the rate of apoptosis in neoplastic cells [49].

\section{PHYSICAL AGENT MODALITIES}

Physical agent modalities, although generally considered adjunctive rather than curative treatments, are widely used and important in the daily practices of most physiatrists [50]. They include cold (cryotherapy), heat (thermotherapy), light (actinotherapy), ultrasound waves, electrical nerve-stimulation, acupuncture.

Cryotherapy- low temperatures will slow down the conduction of nerve stimuli, specifically the myelinated primary efferent nerve fibers $(\mathrm{A}-\delta$ and $\mathrm{C})$, slowing down the local inflammation, and reducing the blood flow through peripheral vasoconstriction. Application of ice slows down of the nerve conduction of small caliber myelinated fibers (Aä and C). Also, it causes peripheral vasoconstriction (and subsequent reduced blood flow) and slows down of local inflammation [51]. Cryotherapy activates transient receptor protein channels (TRP) on afferent nerve endings so that no other noxious stimuli could be transmitted throughout the nociception pathway [52].

Thermotherapy provides analgesia and decreased muscle tonicity. Unlike cryotherapy, thermotherapy increases tissue temperature, blood flow, metabolism, and connective tissue extensibility. Heat therapy is delivered by three mechanisms: conduction, convection, or conversion [53]. The conduction part can be applied by heat packs, or paraffin bath (that induce heat in the body). The convection part is the through fluid therapy or hydrotherapy. And the last mechanism, conversion, can be achieved through ultrasound, heat lamp, or electrical-inducing heat. Thermotherapy leads to an increase in blood flow which will facilitate tissue healing by supplying protein, nutrients and oxygen to the site of injury. A $1^{\circ} \mathrm{C}$ increase in tissue temperature is associated with $10-15 \%$ increase in local tissue metabolism [54]. The application of heat will lead to peripheral vasodilatation and stimulates numerous thermosensitive calcium channel receptors. This will increase intracellular calcium and potentiate the activity of local peripheral sensory nerves in order to transmit to the brain the sensation of heat, meanwhile inhibiting activity of pain receptors [55]. Tissue can be heated up to approximately $1 \mathrm{~cm}$ in depth, for maximum 15 to 20 minutes, one to three sessions per day [51].

Another method involves treatment with rays of light especially ultra-violet or actinic rays, called actinotherapy. These rays can heat up deep seated tissues producing warmth and analgesia. Infrared rays can penetrate epithelium and reach deep tissue to produce warmth and analgesia [56]. However, the duration of therapy should be from fifteen minutes to one hour [5,57]. Further studies are needed in order to establish the best protocols and the treatment opportunity for cancer patients.

Transcutaneous electrical nerve stimulation (TENS) is a very popular method used for pain control. There are two modalities in which the TENS works, gate-control TENS and endorphinic 
TENS. Gate-control TENS acts by determining peripheral hyperstimulation of large caliber sensitive fibers at high-frequency thus inhibiting the transmission of nociceptive influxes conveyed by small-caliber fibers in the dorsal horn of the spinal cord. This type of current generates quick but short-lasting analgesia and is indicated especially for acute pain. The endorphinic TENS works by stimulation of small-caliber fibers at very low frequency which stimulate the release of endorphins. Endorphinic TENS is indicated mainly for chronic pain [58]. In order to be effective, transcutaneous electrical nerve stimulation should be applied for a period of 20 to 30 minutes [51].

Another physical method, which may inhibit pain, is acupuncture. Focal acupoints will activate the local gate-controlled calcium channels and distal acupoints will stimulate the release of endogenous opioids. The analgesic action of acupuncture results from gate-control mechanisms when focal acupoints are treated and from stimulating the release of endogenous opioids when distal spots are treated to procure long term analgesia [58]. For acute pain one session every 2-3 days is necessary until clinical signs are abolished, whereas for chronic pain, multiple sessions are necessary [59]. Acupuncture can be used in combination or independently in the analgesic protocols of cancer patients due to is significant effect on inflammatory, neuropathic, and visceral pain.

An additional form of pain-relieving therapy is hydrotherapy, widely used to improve immunity and for the management of pain at various temperatures, that can produce different effects on different system of the body [60]. Water temperature is an important factor for hydrotherapy for improving immune function. Thermoregulation and immune regulation could represent important strategies for strengthening anti-tumor immunity and needs further researches in both human and veterinary medicine [61].

A method that is clinically supported for treating pain is laser therapy. Laser therapy is an increasingly studied modality that can be a valuable tool for veterinary practitioners, demonstrated to relieve both chronic and acute pain by modulating peripheral nerve function and nerve conduction velocities [62]. Mechanisms of action have been studied and identified for the reduction of pain and inflammation and healing of tissue [62]. The therapy increases the number of endorphins and opioids in the body both locally, and systemically and relieve pain with minimal side effects [63]. Significant applications are developing scientifically: the treatment of internal organ diseases, the regenerative effects on nervous tissue and the possibility of a beneficial cell-specific cytotoxicity are relevant for oncological cases [64].

Laser therapy has the potential to influence pain perception by direct and indirect actions on superficial nociceptors and modulation of inflammation, repeated application of laser therapy may decrease central sensitization. The superficial location of nerve fibers (unmyelinated $\mathrm{C}$ fibers, myelinated $\mathrm{A}-\delta$ fibers), allows laser penetration with consequent inhibitory effects for pain receptors [65].

\section{CONCLUSIONS}

To adequately manage cancer pain in dogs and cats, pain must be recognized early and patients should be frequently assessed for it by both the attending clinician and the pet owner [28]. While pharmacological and physical methods to relief pain develop continuously, it is an imperious need to asses all the newest data and to integrate them in the current practice in order to provide better options for welfare and life quality along with the cancer management. Further researches 
Vol. 06, No. 06; 2021

ISSN: $2456-8643$

need to fill all the gaps in the knowledge regarding assessment and pain management for the veterinary oncological patients.

\section{REFERENCES}

1.Elliott, J., \& Alderson, B. (2019). Managing cancer pain in dogs and cats. In Practice, 41(8), pp. 361-367. https://doi.org/10.1136/inp.15486

2.Pinho SS, Carvalho S, Cabral J, Reis CA, Gartner F. (2012). Canine tumors: a spontaneous animal model of human carcinogenesis. Transl Res 159, pp. 165-72.

3.Raja, S. N., Carr, D. B., Cohen, M., Finnerup, N. B., Flor, H., Gibson, S., ... \& Vader, K. (2020). The revised International Association for the Study of Pain definition of pain: concepts, challenges, and compromises. Pain, 161(9), pp. 1976-1982. doi: 10.1097/j.pain.0000000000001939

4.Lamont, L. A., Tranquilli, W. J., \& Grimm, K. A. (2000). Physiology of pain. Veterinary Clinics: Small Animal Practice, 30(4), pp. 703-728.

5.Dewangan, R., \& Tiwari, S. K. (2019). Physiology of pain and its management in veterinary patients. Pharma Innov, 8, pp. 11-68.

6.Posner, L. P. (2011). Ten Tips to improve Anesthesia in Your Practice. Today's Veterinary Practice, 1(3), pp. 47-51.

7.Banasik, J. L. (2021). Pathophysiology-E-Book. Elsevier Health Sciences, pp. 977.

8.Grimm, K. A., Lamont, L. A., Tranquilli, W. J., Greene, S. A., \& Robertson, S. A. (2015). Veterinary anesthesia and analgesia. John Wiley \& Sons.

9.Chapman, C. R., \& Vierck, C. J. (2017). The transition of acute postoperative pain to chronic pain: an integrative overview of research on mechanisms. The Journal of pain, 18(4), pp.359.

10.Jensen, T. S., Baron, R., Haanpää, M., Kalso, E., Loeser, J. D., Rice, A. S., \& Treede, R. D. (2011). A new definition of neuropathic pain. Pain, 152(10), pp. 2204-2205.

11.Bennett, M. I., Kaasa, S., Barke, A., Korwisi, B., Rief, W., \& Treede, R. D. (2019). The IASP classification of chronic pain for ICD-11: chronic cancer-related pain. Pain, 160(1), pp. 38-44.

12.Scholz, J., Finnerup, N. B., Attal, N., Aziz, Q., Baron, R., Bennett, M. I., ... \& Treede, R. D. (2019). The IASP classification of chronic pain for ICD-11: chronic neuropathic pain. Pain, 160(1), pp. 53.

13.IASP https://www.iasp-pain.org/terminology?navItemNumber=576\#Pain, Accessed 10th April 2021

14.Basbaum AI, Jessel TM. The perception of pain. In: Kandel ER, Schwartz JH, Jessel TM, eds. Principles of Neural Science. New York: McGraw-Hill, pp. 472-491

15.Pinto, L. G., Souza, G. R., Kusuda, R., Lopes, A. H., Sant'Anna, M. B., Cunha, F. Q., \& Cunha, T. M. (2019). Non-peptidergic nociceptive neurons are essential for mechanical inflammatory hypersensitivity in mice. Molecular neurobiology, 56(8), pp. 5715-5728.

16.Yam, M. F., Loh, Y. C., Tan, C. S., Khadijah Adam, S., Abdul Manan, N., \& Basir, R. (2018). General pathways of pain sensation and the major neurotransmitters involved in pain regulation. International journal of molecular sciences, 19(8), pp. 2164.

17.Julius, D., \& Basbaum, A. I. (2001). Molecular mechanisms of nociception. Nature, 413(6852), 203-210.

18.Briggs, E. (2010). Assessment and expression of pain: Pain Series\# 2. Nursing standard, 25(2). 
19.Schmidt, B. L., Hamamoto, D. T., Simone, D. A., \& Wilcox, G. L. (2010). Mechanism of cancer pain. Molecular interventions, 10(3), pp. 164.

20.Fallon, M. T. (2013). Neuropathic pain in cancer. British journal of anaesthesia, 111(1), pp. 105-111. DOI: https://doi.org/10.1093/bja/aet208

21.Portenoy, R. K. (2011). Treatment of cancer pain. The Lancet, 377(9784), pp. 2236-2247.

22.Edwards, H. L., Mulvey, M. R., \& Bennett, M. I. (2019). Cancer-related neuropathic pain. Cancers, 11(3), pp. 373.

23.Marchettini, P., Lacerenza, M., Mauri, E., \& Marangoni, C. (2006). Painful peripheral neuropathies. Current neuropharmacology, 4(3), pp. 175-181.

24.Yoon, S. Y., \& Oh, J. (2018). Neuropathic cancer pain: prevalence, pathophysiology, and management. The Korean journal of internal medicine, 33(6), pp. 1058.

25.Smith, A. N. (2014). Advances in Veterinary Oncology, An Issue of Veterinary Clinics of North America: Small Animal Practice (Vol. 44, No. 5). Elsevier Health Sciences.

26.Self, I., \& Grubb, T. (2019). Physiology of pain. In BSAVA Guide to Pain Management in Small Animal Practice. BSAVA Library. pp 3-13.

27.Flecknell, P. (2008). Analgesia from a veterinary perspective. British journal of anaesthesia, 101(1), pp.121-124.

28.De Lorimier, L. P., \& Fan, T. M. (2005). Understanding and recognizing cancer pain in dogs and cats. Veterinary medicine, 100(5), pp.352-362.

29.Rancilio, N., Poulson, J., \& Ko, J. (2015) Part 1: Pathophysiology \& Assessment of Cancer Pain, pp. 60-67.

30.Kirkpatrick, D. R., McEntire, D. M., Smith, T. A., Dueck, N. P., Kerfeld, M. J., Hambsch, Z. J., \& Agrawal, D. K. (2016). Transmission pathways and mediators as the basis for clinical pharmacology of pain. Expert review of clinical pharmacology, 9(10), pp. 1363-1387.

31.Choi, Y. S., \& Billings, J. A. (2002). Opioid antagonists: a review of their role in palliative care, focusing on use in opioid-related constipation. Journal of pain and symptom management, 24(1), pp. 71-90.

32.Poradowski, D., \& Obmińska-Mrukowicz, B. (2019). Effect of selected nonsteroidal antiinflammatory drugs on the viability of canine osteosarcoma cells of the D-17 line: in vitro studies. Journal of veterinary research, 63(3), pp. 399.

33.Pang, L. Y., Hurst, E. A., \& Argyle, D. J. (2016). Cyclooxygenase-2: a role in cancer stem cell survival and repopulation of cancer cells during therapy. Stem cells international, 2016.

34.Winacoo, J. N., \& Maykel, J. A. (2009). Operative anesthesia and pain control. Clinics in colon and rectal surgery, 22(01), pp. 041-046.

35.Coutinho, A. E., \& Chapman, K. E. (2011). The anti-inflammatory and immunosuppressive effects of glucocorticoids, recent developments and mechanistic insights. Molecular and cellular endocrinology, 335(1), pp. 2-13.

36.Pufall, M. A. (2015). Glucocorticoids and cancer. Glucocorticoid Signaling, pp. 315-333.

37.Hansen, K. B., Yi, F., Perszyk, R. E., Menniti, F. S., \& Traynelis, S. F. (2017). NMDA receptors in the central nervous system. In NMDA Receptors Humana Press, New York, NY. pp. $1-80$.

38.Blanpied, T. A., Clarke, R. J., \& Johnson, J. W. (2005). Amantadine inhibits NMDA receptors by accelerating channel closure during channel block. Journal of Neuroscience, 25 (13), pp. 3312-3322. 
39.Sunilchandra, U., Ravikumar, C., \& Rashmi, R. (2020). Analgesics in animal pain management.

40.Bannister, K., Qu, C., Navratilova, E., Oyarzo, J., Xie, J. Y., King, T., \& Porreca, F. (2017). Multiple sites and actions of gabapentin-induced relief of ongoing experimental neuropathic pain. Pain, 158(12), pp. 2386.

41.Giovannitti Jr, J. A., Thoms, S. M., \& Crawford, J. J. (2015). Alpha-2 adrenergic receptor agonists: a review of current clinical applications. Anesthesia progress, 62(1), pp. 31-38.

42.Flecknell, P., Lofgren, J. L., Dyson, M. C., Marini, R. R., Swindle, M. M., \& Wilson, R. P. (2015). Preanesthesia, anesthesia, analgesia, and euthanasia. In Laboratory animal medicine. Academic Press. pp. 1135-1200.

43.Gaynor JS, Muir WW. (2002). Handbook of veterinary pain management. St. Louis, Mo: Mosby, pp. 14-15.

44.Taylor, A., \& McLeod, G. (2020). Basic pharmacology of local anaesthetics. BJA education, 20(2), pp. 34-41.

45.Barreveld, A., Witte, J., Chahal, H., Durieux, M. E., \& Strichartz, G. (2013). Preventive analgesia by local anesthetics: the reduction of postoperative pain by peripheral nerve blocks and intravenous drugs. Anesthesia and analgesia, 116(5), pp. 1141.

46Katz, J., Clarke, H., \& Seltzer, Z. E. (2011). Preventive analgesia: quo vadimus? Anesthesia \& Analgesia, 113(5), pp. 1242-1253.

47.Patrick, D. L., Cleeland, C. S., von Moos, R., Fallowfield, L., Wei, R., Öhrling, K., \& Qian, Y. (2015). Pain outcomes in patients with bone metastases from advanced cancer: assessment and management with bone-targeting agents. Supportive Care in Cancer, 23(4), pp. 1157-1168. 48.Taverner, T. (2015). Neuropathic pain in people with cancer (part 2): pharmacological and non-pharmacological management. International journal of palliative nursing, 21(8), pp. 380384.

49.Pertwee, R. G. (2012). Targeting the endocannabinoid system with cannabinoid receptor agonists: pharmacological strategies and therapeutic possibilities. Philosophical Transactions of the Royal Society B: Biological Sciences, 367(1607), pp. 3353-3363.

50.Chen, W. S., Annaswamy, T. M., Yang, W., Wang, T. G., Kwon, D. R., \& Chou, L. W. (2021). Physical agent modalities. In Braddom's physical medicine and rehabilitation Elsevier. pp. 338-363.

51.Sawaya, S. (2007). Physical and alternative therapies in management of arthritic patients. Vet. Foc. 17(3): pp. 37-42.

52.Emir, T. L. R. (Ed.). (2017). Neurobiology of TRP channels. pp. 127-128

53.Nadler, S. F., Weingand, K., \& Kruse, R. J. (2004). The physiologic basis and clinical applications of cryotherapy and thermotherapy for the pain practitioner. Pain physician, 7(3), pp. 395-400.

54.Sturesson, C. (1998). Medical laser-induced thermotherapy-models and applications. Lund University.

55.Vriens, J., Nilius, B., \& Voets, T. (2014). Peripheral thermosensation in mammals. Nature Reviews Neuroscience, 15(9), pp. 573-589.

56.Kumar, S. P., \& Jim, A. (2010). Physical therapy in palliative care: from symptom control to quality of life: a critical review. Indian journal of palliative care, 16(3), pp. 138. 
Vol. 06, No. 06; 2021

ISSN: $2456-8643$

57.Venugopalan, A. (2005). Counter-irritation; physiotherapy and actinotherapy. Essentials of veterinary surgery. 8th Ed. Oxford and IBH Publishing, New Delhi. Pp. 125-129

58.Vedpathak, H. S., Tank, P. H., Karle, A. S., Mahida, H. K., Joshi, D. O., \& Dhami, M. A. (2009). Pain Management in Veterinary Patients. Veterinary World, 2(9).

59.Altman, S. (1998). Small animal acupuncture: Scientific basis and clinical applications. Complementary and alternative veterinary medicine: Principles and practice by Shoen, AM and Wynn, SG Mosby Publications, USA, pp. 147-167.

60.Mooventhan, A., \& Nivethitha, L. (2014). Scientific evidence-based effects of hydrotherapy on various systems of the body. North American journal of medical sciences, 6(5), pp. 199.

61.Lee, B., Kim, G., Jo, Y., Lee, B., Shin, Y. I., \& Hong, C. (2019). Aquatic exercise at thermoneutral water temperature enhances antitumor immune responses. Immune network, 19(2). DOI: https://doi.org/10.4110/in.2019.19.e10

62.Pryor, B., \& Millis, D. L. (2015). Therapeutic laser in veterinary medicine. Veterinary Clinics: Small Animal Practice, 45(1), pp. 45-56.

63.Hagiwara, S., Iwasaka, H., Okuda, K., \& Noguchi, T. (2007). GaAlAs (830 nm) low-level laser enhances peripheral endogenous opioid analgesia in rats. Lasers in Surgery and Medicine: The Official Journal of the American Society for Laser Medicine and Surgery, 39(10), pp. 797802.

64.Paterniani, V., \& Grolli, S. (2018, April). Approach and potentiality of low-level laser therapy in veterinary medicine. In Laser Florence 2017: Advances in Laser Medicine (Vol. 10582, p. 1058205). International Society for Optics and Photonics.

65. Millis, D. L., Francis, D., \& Adamson, C. (2005). Emerging modalities in veterinary rehabilitation. Veterinary Clinics: Small Animal Practice, 35(6), pp. 1335-1355. 